\title{
Role of spinal LPCAT2, an inducible PAF synthesis enzyme on development and maintenance of painful peripheral neuropathy models in mice
}

\author{
Katsuya Morita ${ }^{1}$, Naoyo Motoyama ${ }^{2}$, Tomoya Kitayama ${ }^{3}$, Mari Sanai ${ }^{4}$, Midori Hashimoto ${ }^{5}$, \\ Akiko Tsuchii ${ }^{1}$, Kazuna Kato ${ }^{1}$, Yuno Kawai ${ }^{1}$, Toshihiro Dohi ${ }^{1}$ \\ ${ }^{\text {I} P h a r m a c o l o g y, ~ H i r o s h i m a ~ B u n k a ~ G a k u e n ~ U n i v e r s i t y, ~ J a p a n, ~}{ }^{2}$ Biological Endodontics, Hiroshima University, Japan, \\ ${ }^{3}$ Pharmacy, Mukogawa Women's University, Japan, ${ }^{4}$ Gerontology, Hiroshima Bunka Gakuen University, Japan, \\ ${ }^{5}$ Psychology, Hiroshima Bunka Gakuen University, Japan
}

Pain in peripheral neuropathies is produced by damage or illness affecting nerve fibers themselves, and often resistant to current analgesics. We have previously suggested that PAF may be a mediator of neuropathic pain. PAF injection into the mouse spinal cord caused thermal hyperalgesia and tactile allodynia, which were at least in part mediated by spinal dysfunction of glycine receptor a3 (GlyRa3) and were blocked by PAF receptor antagonists [Morita et al., Pain 111:351 -359,2004; Pain 138: 525-536,2008]. Subsequent studies showed PAF receptor blockade reduced pain behaviors elicited in nerve injury models [Okubo et al., Mol Pain 8(8):doi:10.1186,2012; Motoyama et al., Eur J Pain 17(8):1156 -1167,2013; Morita et al., PlosOne 9(3):e91746,2014].

The present study examined the pain relieving effect of knockdown of spinal LPCAT2, a novel inducible PAF synthesis enzyme, and of specific inhibitor of LPCAT2 (TSI-01) on several neuropathic pain models (partial sciatic nerve ligationinjury model, painful diabetic neuropathy model, bone cancer pain model, chemotherapeutic agents-induced painful neuropathy, chronic and acute inflammatory pain models in animal).

This study demonstrated that the amount of a LPCAT2 protein significantly increased in the spinal cord after sciatic nerve ligation and transplantation of NCTC 2472 tumor cells into mouse tibia [Morita et al., PlosOne 9(3):e91746,2014]. The knockdown of spinal LPCAT2 and PAF receptor by intrathecal small interfering RNA (siRNA) transfer 15 days after sciatic nerve injury ameliorated tactile allodynia. Knockdown of LPCAT2 and PAF receptor 3 day before sciatic nerve injury prevented the development of pain until 130 days after surgery. Single administration of TSI-01 and repeated administration of specific PAF receptor antagonist (TCV-309) abolished the appearance of pain behaviors at pre- and post-surgery in the partial sciatic nerve ligation injury model, respectively. The anti-nociceptive effects of knockdown of LPCAT2 and PAF receptor, repeated administration of PAF antagonist TCV-309 and LPCAT2 inhibitor TSI-01 was appeared certainly in any other models tested.

These data suggest that spinal LPCAT2 play a causal role in the development and maintenance of neuropathic pain in animal models, and that LPCAT2 antagonists may highlight a potential novel therapeutic strategy for the treatment of persistent neuropathic pain. 